Lei Fang, Panos J. Antsaklis, "On Communication Requirements for Multi-agent Consensus Seeking,"

Networked Embedded Sensing and Control, Proceedings of Workshop NESC'05: University of Notre Dame, USA, October 17-18, 2005, Panos Antsaklis and Paulo Tabuada (Eds.), Lecture Notes in Control and Information Sciences (LNCIS) 331, pp. 53-68, Springer 2006.

\title{
On Communication Requirements for Multi-agent Consensus Seeking
}

\author{
Lei Fang and Panos J. Antsaklis \\ Department of Electrical Engineering, University of Notre Dame, Notre Dame, IN \\ 46556, USA, \{lfang, antsaklis.1\}@nd.edu
}

Several consensus protocols have been proposed in the literature and their convergence properties studied via a variety of methods. In all these methods, the communication topologies play a key role in the convergence of consensus processes. The goal of this paper is two fold. First, we explore communication topologies, as implied by the communication assumptions, that lead to consensus among agents. For this, several important results in the literature are examined and the focus is on different classes of communication assumptions being made, such as synchronism, connectivity, and direction of communication. In the latter part of this paper, we show that the confluent iteration graph unifies various communication assumptions and proves to be fundamental in understanding the convergence of consensus processes. In particular, based on asynchronous iteration methods for nonlinear paracontractions, we establish a new result which shows that consensus is reachable under directional, time-varying and asynchronous topologies with nonlinear protocols. This result extends the existing ones in the literature and have many potential applications.

\section{Introduction}

In recent years, there has been growing interest in the coordinated control of multi-agent systems. One of the fundamental problems is the consensus seeking among agents, that is the convergence of the values of variables common among agents to the same constant value $[2,17,21,26,28]$. This need stems from the fact that in order for agents to coordinate their behaviors, they need to use some shared knowledge about variables such as direction, speed, time-to-target etc. This shared variable or information is a necessary condition for cooperation in multi-agent systems, as shown in [30]. The challenge here is for the group to have a consistent view of the coordination variable in the presence of unreliable and dynamically changing communication topology 
without global information exchange. For an extensive body of related work, see $[2,1,10,15,19,21,29,31,35]$.

The aforementioned consensus protocols all operate in a synchronized fashion since each agent's decisions must be synchronized to a common clock shared by all other agents in the group. This synchronization requirement might not be natural in certain contexts. For example, the agreement of timeon-target in cooperative attack among a group of UAVs depends in turn on the timing of when to exchange and update the local information. This difficulty entails the consideration of the asynchronous consensus problem, where each agent updates on its own pace, and uses the most recently received (but possibly outdated) information from other agents. No assumption is made about the relative speeds and phases of different clocks. Agents communicate solely by sending messages; however there is no guarantee of the time of delivery or even for a successful delivery. Under asynchronous communications, heterogeneous agents, time-varying communication delays and packet dropout can all be taken into account in the same framework. Nevertheless, the asynchronism can destroy convergence properties that the algorithm may possess when executed synchronously. In general, the analysis of asynchronous algorithms is more difficult than that of their synchronous counterparts. We refer readers to $[5,14,18]$ for surveys on general theories of asynchronous systems.

Work reported on the asynchronous consensus problem is relatively sparse compared to its synchronous counterparts. In [12], we introduced an asynchronous framework to study the consensus problems for discrete-time multiagent systems with a fixed communication topology under the spanning tree assumption (All the assumptions in this paragraph will be discussed in detail later). A distributed iterative procedure under the eventual update assumption was developed in [23] for calculating averages on asynchronous communication networks. The asynchronous consensus problem with zero time delay was studied in [8] where the union of the communication graphs is assumed to have a common root spanning tree. A nice overview of the asynchronous consensus problem is given in [6] where the authors link the consensus problem considered here to earlier work [33]. For other related problems in asynchronous multi-agent systems, see $[3,20,22,34]$.

Asynchronism provides a new dimension to consensus problems and makes convergence harder to achieve. Under certain technical conditions, asynchronism is not detrimental to consensus seeking among agents. A natural question is what are the appropriate requirements on communication topologies to guarantee the convergence of consensus processes? In order to answer this question, we first discuss the various assumptions on communication topologies commonly used in the literature and classify several of the existing consensus results by these communication assumptions. In Sect. 3, we prove the convergence of asynchronous consensus with zero time delay involving pseudocontractive mappings. This development is a generalization of the results of [8] and [36], and provides insight into why the choice between bidirectional and unidirectional communication assumptions can make the difference in es- 
tablishing consensus convergence. In Sect. 4 , we unify various communication assumptions using the confluent iteration graph proposed in [27]. Furthermore, a new convergence result for nonlinear protocols is developed based on the confluent asynchronous iteration concept. This result contains some existing ones in $[6]$ as special cases.

\section{Preliminaries and Background}

\subsection{Definitions and Notations}

Let $G=\{V, E, A\}$ be a weighted digraph (or direct graph) of order $n$ with the set of nodes $V=\left\{v_{1}, v_{2}, \ldots, v_{n}\right\}$, set of edges $E \subseteq V \times V$, and a weighted adjacency matrix $A=\left[a_{i j}\right]$ with nonnegative adjacency elements $a_{i j}$. The node indices belong to a finite index set $\mathcal{I}=\{1,2, \ldots, n\}$. A directed edge of $G$ is denoted by $e_{i j}=\left(v_{i}, v_{j}\right)$. For a digraph, $e_{i j} \in E$ does not imply $e_{j i} \in E$. The adjacency elements associated with the edges of the graph are positive, i.e., $a_{i j}>0$ if and only if $e_{j i} \in E$. Moreover, we assume $a_{i i} \neq 0$ for all $i \in \mathcal{I}$. The set of neighbors of node $v_{i}$ is the set of all nodes which point to (communicate with) $v_{i}$, denoted by $N_{i}=\left\{v_{j} \in V:\left(v_{j}, v_{i}\right) \in E\right\}$.

A digraph $G$ can be used to model the interaction topology among a group of agents, where every graph node corresponds to an agent and a directed edge $e_{i j}$ represents a unidirectional information exchange link from $v_{i}$ to $v_{j}$, that is, agent $j$ can receive information from agent $i$. The interaction graph represents the communication pattern at certain time. The interaction graph is time-dependent since the information flow among agents may be dynamically changing. Let $\bar{G}=\left\{G_{1}, G_{2}, \ldots, G_{M}\right\}$ denote the set of all possible interaction graphs defined for a group of agents. Note that the cardinality of $\bar{G}$ is assumed to be finite. The union of a collection of graphs $\left\{G_{i_{1}}, G_{i_{2}}, \ldots, G_{i_{m}}\right\}$, each with the vertex set $V$, is a graph $\mathbb{G}$ with the vertex set $V$ and the edge set equal to the union of the edge sets of $G_{i_{j}}, j=1, \ldots, m$.

A directed path in graph $G$ is a sequence of edges $e_{i_{1} i_{2}}, e_{i_{2} i_{3}}, e_{i_{3} i_{4}}, \cdots$ in that graph. Graph $G$ is called strongly connected if there is a directed path from $v_{i}$ to $v_{j}$ and $v_{j}$ to $v_{i}$ between any pair of distinct vertices $v_{i}$ and $v_{j}$. Vertex $v_{i}$ is said to be linked to vertex $v_{j}$ across a time interval if there exists a directed path from $v_{i}$ to $v_{j}$ in the union of interaction graphs in that interval. A directed tree is a directed graph where every node except the root has exactly one parent. A spanning tree of a directed graph is a tree formed by graph edges that connect all the vertices of the graph. The condition that a digraph contains a spanning tree is equivalent to the condition that there exists a node having a directed path to all other nodes.

Let $x_{i} \in \mathbb{R}, i \in \mathcal{I}$ represent the state associated with agent $i$. A group of agents is said to achieve global consensus asymptotically if for any $x_{i}(0)$, $i \in \mathcal{I},\left\|x_{i}(t)-x_{j}(t)\right\| \rightarrow 0$ as $t \rightarrow \infty$ for each $(i, j) \in \mathcal{I}$. Let $\mathbf{1}$ denote an $n \times 1$ column vector with all entries equal to 1 . A matrix $F \in \mathbb{R}^{n \times n}$ is nonnegative, 
$F \geq 0$, if all its entries are nonnegative, and it is irreducible if and only if $(I+|F|)^{n-1}>0$, where $|F|$ denotes the matrix of absolute values of entries of $F$. Furthermore, if all its row sums are $+1, F$ is said to be a (row) stochastic matrix.

Let $X^{*}$ be a nonempty closed convex subset of $\mathbb{R}^{n}$, and let $\|\cdot\|$ be a norm on $\mathbb{R}^{n}$. For any vector $x \in \mathbb{R}^{n}, y^{*} \in X^{*}$ is a projection vector of $x$ onto $X^{*}$ if $\left\|x-y^{*}\right\|=\min _{y \in X^{*}}\|x-y\|$. We use $P(x)$ to denote an arbitrary but fixed projection vector of $x$ and $\operatorname{dist}\left(x, X^{*}\right)$ to denote $\|x-P(x)\|$. Let $T$ be an operator on $\mathbb{R}^{n}$. It is paracontractive if

$$
\|T x\| \leq\|x\| \text { for all } x \in \mathbb{R}^{n}
$$

and equality holds if and only if $T x=x$. An operator is nonexpansive (with respect to $\|\cdot\|$ and $X^{*}$ ) if

$$
\left\|T x-x^{*}\right\| \leq\left\|x-x^{*}\right\| \text { for all } x \in \mathbb{R}^{n}, x^{*} \in X^{*},
$$

and pseudocontractive [32] (with respect to $\|\cdot\|$ and $X^{*}$ ) if, in addition,

$$
\operatorname{dist}\left(T x, X^{*}\right)<\operatorname{dist}\left(x, X^{*}\right) \text { for all } x \notin X^{*} .
$$

We use $\mathcal{T}$ to denote the set of all pseudocontractive operators. In the linear case, pseudocontractive operators are generalizations of paracontractive ones. But the converse is not true. Consider the following inequalities:

$$
\|T x-P(T x)\| \leq\|T x-P(x)\| \leq\|x-P(x)\| \text {, for all } x \notin X^{*} .
$$

Paracontractivity requires the second inequality to be strict, while pseudocontractivity requires any one of these two inequalities to be strict.

Example 1. Let $T \in \mathbb{R}^{n \times n}, X^{*}=\{c \mathbf{1} \mid c \in \mathbb{R}\}$. Then $T$ is pseudocontractive with respect to $X^{*}$ and the infinity norm $\|\cdot\|_{\infty}$ if and only if $T \mathbf{1}=\mathbf{1}$ and for any $x \in \mathbb{R}^{n}$ such that $\min _{i} x_{i}<\max _{i} x_{i}, \max _{i}(T x)_{i}-\min _{i}(T x)_{i}<\max _{i} x_{i}-$ $\min _{i} x_{i}$.

\subsection{Synchronous and Asynchronous Consensus Protocols}

We consider the following (synchronous) discrete-time consensus protocol [28, $26,24]$

$$
x_{i}(t+1)=\frac{1}{\sum_{j=1}^{n} a_{i j}(t)} \sum_{j=1}^{n} a_{i j}(t) x_{j}(t)
$$

where $t \in\{0,1,2, \cdots\}$ is the discrete-time index, $(i, j) \in \mathcal{I}$ and $a_{i j}(t)>0$ if information flows from $v_{j}$ to $v_{i}$ at time $t$ and zero otherwise, $\forall j \neq i$. The magnitude of $a_{i j}(t)$ possibly represents time-varying relative confidence of agent $i$ in the information state of agent $j$ at time $t$ or the relative reliabilities 
of information exchange links between agents $i$ and $j$. We can rewrite (5) in a compact form

$$
x(t+1)=F(t) x(t)
$$

where $x=\left[x_{1}, \cdots, x_{n}\right]^{T}, F(t)=\left[F_{i j}(t)\right]$ with $F_{i j}(t)=\frac{a_{i j}(t)}{\sum_{j=1}^{n} a_{i j}(t)},(i, j) \in \mathcal{I}$. An immediate observation is that the matrix $F$ is a nonnegative stochastic matrix, which has an eigenvalue at 1 with the corresponding eigenvector equal to 1 . The protocol (5) or (6) is synchronous in the sense that all the agents update their states at the same time using the latest values of neighbors' states.

The way described above to define $F(t)$ in (6) is only one possible way among many others. In the following we assume that $F(t)$ satisfies Assumption 1 below.

Assumption 1 (Nontrivial interaction strength [6]) There exists a positive constant $\alpha$ such that

(a) $F_{i i}(t) \geq \alpha$, for all $i, t$.

(b) $F_{i j}(t) \in\{0\} \cup[\alpha, 1]$, for all $i, j, t$.

(c) $\sum_{j=1}^{n} F_{i j}(t)=1$, for all $i, t$.

Now, in the asynchronous setting the order in which states of agents are updated is not fixed and the selection of previous values of the states used in the updates is also not fixed. Now let $t_{0}<t_{1}<\cdots<t_{n}<\cdots$ be the time instants when the state of the multi-agent system undergoes change. Let $x_{i}(k)$ denote the state of agent $i$ at time $t_{k}$. The index $k$ is also called in the literature the event-based discrete time index. The dynamics of asynchronous systems can be written as

$$
x_{i}(k+1)= \begin{cases}\sum_{j=1}^{n} F_{i j}(k) x_{j}\left(s_{j}^{i}(k)\right) & \text { if } i \in I(k), \\ x_{i}(k) & \text { if } i \notin I(k),\end{cases}
$$

where $s_{j}^{i}(k)$ are nonnegative integers, $I(k)$ are nonempty subsets of $\{1, \cdots, n\}$. The initial states are specified by $x(0)=x(-1)=\cdots$. Henceforth, we write the initial vector $x(0)$ to abbreviate reference to this set of equal initial states. We refer to $d_{j}^{i}(k)=k-s_{j}^{i}(k)$ as iteration delays and $I(k)$ as updating sets. The following assumptions (called regularity assumptions) are usually made in the study of linear asynchronous linear systems.

Assumption 2 (Partial asynchronism) (a) (Frequency of updating) The updating sets $I(k)$ satisfy

$$
\exists B \geq 0, \bigcup_{k=i}^{i+B} I(k)=\{1, \cdots, n\}, \text { for all } i .
$$


(b) (Bounded-delay asynchronism) There exists a nonnegative integer B such that

$$
0 \leq k-s_{j}^{i}(k) \leq B<\infty, \forall(i, j, k) .
$$

(c) $s_{i}^{i}(k)=k$, for all $i$.

Assumption 2(a) says that every agent should be updated at least once in any $B+1$ iteration steps. Assumption 2(b) requires delays to be bounded by some constant $B$. Assumption 2(c) says that an agent generally has access to its own most recent value. Without loss of generality (but after renumbering in the original definition), we assume that $I(k)$ is a singleton which contains a single element from $\{1, \ldots, n\}$. Furthermore, if for all $i$ there exists a nonnegative integer $B_{i}$ such that for all $j$

$$
i \in \bigcup_{k=j}^{j+B_{i}} I(k)
$$

we call $I(k)$ an indexwise-regulated sequence [27]. This condition expresses the fact that different agents may have different updating frequencies.

\subsection{Other Communication Assumptions}

Let us review several important assumptions commonly used in the literature.

Assumption 3 (Connectivity) (a) (Uniform strong connectivity) There exists a nonnegative integer $B$ such that $\bigcup_{s=t}^{t+B} G(s)$ is strongly connected for all $t$.

(a') (Uniform spanning tree) There exists a nonnegative integer B such that $\bigcup_{s=t}^{t+B} G(s)$ contains a spanning tree for all $t$.

(b) (Nonuniform strong connectivity) $\bigcup_{t \geq t_{0}} G(t)$ is strongly connected for all $t_{0} \geq 0$.

(b') (Nonuniform spanning tree) $\bigcup_{t \geq t_{0}} G(t)$ contains a spanning tree for all $t_{0} \geq 0$.

\section{Assumption 4 (Direction of Communication)}

(a) (Bidirectional link) If $e_{i j} \in G(t)$ then $e_{j i} \in G(t)$. It implies that the updating matrix $F(t)$ or $F(k)$ is symmetric.

(b) (Unidirectional link) $e_{i j} \in G(t)$ does not imply $e_{j i} \in G(t)$. In this case, the updating matrix $F(t)$ or $F(k)$ is not symmetric.

\section{Assumption 5 (Reversal link)}

(a) If $\left(v_{i}, v_{j}\right) \in G(t)$, then there exists some $\tau$ such that $|t-\tau|<B$ and $\left(v_{j}, v_{i}\right) \in G(\tau)[8,6]$. 
(b) There is a nonnegative integer $B$ such that for all $t$ and all $v_{i}, v_{j} \in V$ we have that if $\left(v_{i}, v_{j}\right) \in G(t)$ then $v_{j}$ is linked to $v_{i}$ across $[t, t+B][25]$.

Assumptions 1-5 play different roles in proving various consensus results and they are not necessarily independent from each other. Assumption 1 (Nontrivial interaction strength) and one of the items in Assumption 3 (Connectivity) are always necessary for the convergence of consensus protocols. They guarantee that any update by any agent has a lasting effect on the states of all other agents. Assumption 2 (Partial asynchronism) describes a class of asynchronous systems. If Assumption 3(a) (Uniform strong connectivity) is satisfied, then Assumption 2(a) (Frequency of Updating) and Assumption 5(b) (Reversal link) are satisfied automatically. Assumption 4(a) (Bidirectional link) is a special case of Assumption 5 (Reversal link). Instead of requiring an instantaneous reversal link $\left(v_{j}, v_{i}\right)$ ( $T=0$ for the bidirectional case) for the link $\left(v_{i}, v_{j}\right)$, we only need the reversal link $\left(v_{j}, v_{i}\right)$ to appear within a certain time window or just require $v_{j}$ links back to $v_{i}$ within a certain time window (The edge $\left(v_{j}, v_{i}\right)$ may not appear at all).

\subsection{A Classification of Consensus Results}

For better understanding of Assumptions 1-5, we categorize some of the existing consensus results in Table 1. Assumption 1 is omitted in the table since it is required in all the results listed.

Table 1. A Categorization of the existing consensus results

\begin{tabular}{c|c|c|c|c|c}
\hline No. & Results & Synchronism & Connectivity & Direction & Reversal link \\
\hline \hline 1 & Th. 2 in $[17]$ & Sync. & A3(a) & A4(a) & NA \\
\hline 2 & Prop. 2 in [24] & Sync. & A3(b) & A4(a) & NA \\
\hline 3 & Prop. 1 in $[24]$ & Sync. & A3(a') & A4(b) & NA \\
\hline 4 & Th. 3.10 in [28] & Sync. & A3(a') & A4(b) & NA \\
\hline 5 & Th. $1[25]$ & Sync. & A3(b) & A4(b) & A5(b) \\
\hline 6 & Th. 2 in [12] & A2(a),(b),(c) & Fixed spanning tree & A4(b) & NA \\
\hline 7 & Th. 4 in $[8]$ & $\begin{array}{c}\text { A2(a),(c) with } \\
\text { zero time delay }\end{array}$ & $\begin{array}{c}\text { Spanning trees with } \\
\text { the common root }\end{array}$ & A4(b) & NA \\
\hline 8 & Th. 1 in $[6]$ & $\begin{array}{c}\text { A2(a),(c) with } \\
\text { zero time delay }\end{array}$ & A3(a) & A4(b) & NA \\
\hline 9 & Th. 3 in $[6]$ & A2(a),(b),(c) & A3(a) & A4(b) & NA \\
\hline 10 & Th. 4 in $[6]$ & A2(a),(b),(c) & A3(b) & A4(a) & NA \\
\hline 11 & Th. 5 in $[6]$ & A2(a),(b),(c) & A3(b) & A4(b) & A5(a) \\
\hline
\end{tabular}

Several comments are appropriate. First, asynchronous systems with zero time delay can be mapped to equivalent synchronous systems following the arguments in [12]. Therefore, Results No. 3 \& 4 may be seen as a special case of Result No. 6. Second, Table 1 reveals the fact that the uniform connectivity 
is necessary under unidirectional communication but it is not necessary under bidirectional communication. The reason behind this fact is not at all clear from an intuitive perspective. In Sect. 3, we give an explanation to the difference between bidirectional and unidirectional communication from contractive operators' point of view. Third, we utilize the iteration graph in Sect. 4.1 to unify various communication assumptions. Fourth, Result No. 9 considers the most general case among linear protocols; notice that all protocols in Table 1 are linear protocols. A nonlinear asynchronous protocol will be introduced in Sect. 4.2 .

\section{Bidirectional vs. Unidirectional Communication}

In this section, we investigate why different assumptions on connectivity need to be imposed for bidirectional and unidirectional communication if consensus is to be achieved. Specifically, we see the consensus problem as a matrix iteration problem where the notions of paracontraction and pseudocontraction introduced in Sect. 2.1 are useful in proving convergence.

For an easy exposition, we restrict ourselves to synchronous protocols with time-varying topologies and no time delays. That is, we consider the system updating equation

$$
x(t+1)=F(t) x(t)
$$

where the matrix $F(t)$ satisfies Assumption 1. It is easily deduced that $F(t)$ is nonexpansive with respect to the vector norm $\|\cdot\|_{\infty}$. In the bidirectional case, $F(t)$ is also symmetric. From nonnegative matrix theory [16], we know that the eigenvalues of $F(t)$ lie in $(-1,+1$ ] for all $t$. It is now known that any symmetric matrix $F$ the eigenvalues of which are in $(-1,+1]$ is paracontracting with respect to Euclidian norm [7]. However, $F(t)$ is no longer paracontracting when its symmetry is lost.

Example 2 (partly taken from [32]) (a) For the weight matrix

$$
F_{1}=\left[\begin{array}{ccc}
0.5 & 0.5 & 0 \\
0.25 & 0.5 & 0.25 \\
0 & 0.5 & 0.5
\end{array}\right]
$$

induced from the simple communication topology as shown in Fig. 1(a), the norm and the set $X^{*}$ are the same as in Example 1. For any $x, P(x)=$ $0.5\left(\max _{i} x_{i}+\min _{i} x_{i}\right) \mathbf{1}$. For $x=[2,2,1]^{T}, F_{1} x=(2,1.75,1.5)^{T}, P(x)=1.5 \cdot \mathbf{1}$, and $P\left(F_{1} x\right)=1.75 \cdot 1$. Thus the first inequality in (4) is strict while the second one is an equality. So this operator is pseudocontractive, not paracontractive.

(b) For an arbitrary $F(t)$, there is no guarantee that it is pseudocontractive, e.g., the weight matrix

$$
F_{2}=\left[\begin{array}{ccc}
1 & 0 & 0 \\
0.5 & 0.5 & 0 \\
0 & 0 & 1
\end{array}\right],
$$


which results from the communication topology in Fig. 1(b). For $x=[2,2,1]^{T}$, $F_{2} x=[2,2,1]^{T}$. Thus equalities hold throughout $(4) . F_{2}$ is not pseudocontractive but it is nonexpansive.

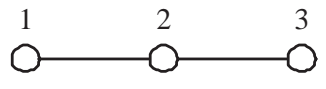

(a)

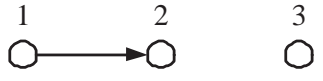

(b)

Fig. 1. Possible communication topologies for a three-agent system

An alternative proof for Result No. 2 was provided in [36] where the convergence of the paracontracting matrix products was explored. The following theorem in [11] is the key to this development.

Theorem 1. Suppose that a finite set of square matrices of same dimensions $\left\{F_{1}, \cdots, F_{r}\right\}$ are paracontracting. Let $\{I(t)\}_{t=0}^{\infty}$, with $1 \leq I(t) \leq r$, be a sequence of integers, and let $\mathcal{J}$ denote the set of all integers that appear infinitely often in the sequence. Then for all $x(0) \in \mathbb{R}^{n}$ the sequence of vectors $x(t+1)=F_{I(t)} x(t)$ has a limit $x^{*} \in \cap_{i \in \mathcal{J}} H\left(F_{i}\right)$, where $H(F)$ denotes the fixed point subspace of a paracontracting matrix $F$, i.e., its eigenspace associated with the eigenvalue $\mathbf{1}$,

$$
H(F)=\left\{x \mid x \in \mathbb{R}^{n}, F x=x\right\} .
$$

Does there exist a result similar to Theorem 1 for pseudocontracting matrices? The answer is, fortunately, affirmative.

Theorem 2 ([32]). Let $\left\{T_{t}\right\}$ be a sequence of nonexpansive operators (with respect to $\|\cdot\|$ and $\left.X^{*}\right)$, and assume there exists a subsequence $\left\{T_{t_{i}}\right\}$ which converges to $T \in \mathcal{T}$. If $T$ is pseudocontractive and uniformly Lipschitz continuous, then for any initial vector $x(0)$, the sequence of vectors $x(t+1)=T_{t} x(t)$, $t \geq 0$ converges to some $x^{*} \in X^{*}$.

Note that in our study $X^{*}=\{c \mathbf{1} \mid c \in \mathbb{R}\}$. We are thus one step away from proving Result No. 3 and it is exactly where Assumption A3(a) (the uniform connectivity) comes into play. Result No. 3 is an immediate result of Theorem 2 and the following lemma.

Lemma 1. If Assumption $A 3(a)$ is satisfied, then there exists an integer $B^{\prime}$ such that the matrix product $F\left(t_{0}+B^{\prime}\right) F\left(t_{0}+B^{\prime}-1\right) \cdots F\left(t_{0}\right)$ is pseudocontractive, with respective to $\|\cdot\|_{\infty}$ and $X^{*}=\{c \mathbf{l} \mid c \in \mathbb{R}\}$, for any $t_{0} \geq 0$.

To prove Lemma 1, we need the following technical results.

Lemma 2 (Lemma 2 in [17]). Let $m \geq 2$ be a positive integer and let $F_{1}, F_{2} \ldots, F_{m}$ be nonnegative $n \times n$ matrices. Suppose that the diagonal 
elements of all of the $F_{i}$ are positive and let $\alpha$ and $\beta$ denote the smallest and largest of these, respectively. Then

$$
F_{m} F_{m-1} \cdots F_{1} \geq\left(\frac{\alpha^{2}}{2 \beta}\right)^{(m-1)}\left(F_{m}+F_{m-1}+\cdots+F_{1}\right) .
$$

Lemma 3 (Proposition 3.2 in [32]). Let $x$ be a vector such that $\underline{x}<\bar{x}$ with $\underline{x}=\min _{i} x_{i}$ and $\bar{x}=\max _{i} x_{i}, F$ be an irreducible matrix with $F_{i i}>0$ for $1 \leq$ $i \leq n, y=F x$. Then the number of the elements in set $\left\{i \mid y_{i}=\underline{x}\right.$ or $\left.y_{i}=\bar{x}\right\}$ is at least one less than the number of the elements in $\left\{i \mid x_{i}=\underline{x}\right.$ or $\left.x_{i}=\bar{x}\right\}$.

Proof (of Lemma 1). Assume that there exists an infinite sequence of contiguous, non-empty, bounded time-intervals $\left[t_{i_{j}}, t_{i_{j+1}}\right), j \geq 1$, starting at $t_{i_{1}}$, with the property that across each such interval, the union of the interaction graphs is strongly connected (Assumption A3(a)).

Let $H_{t_{j}}=F\left(t_{i_{j+1}-1}\right) \cdots F\left(t_{i_{j}+1}\right) F\left(t_{i_{j}}\right)$. By Lemma 2 and the strong connectivity of the union of the interaction graphs, it follows that $H_{t_{j}}$ is irreducible. It is easy to prove that $H_{t_{j}}$ is nonexpansive with positive diagonal elements and $H_{t_{j}} \mathbf{1}=\mathbf{1}$.

Define $H=H_{t_{n-1}} H_{t_{n-2}} \cdots H_{t_{1}}$ and $y=H x$. Applying Lemma 3 repeatedly for $(n-1)$ times, we have at least one set of $\left\{i \mid y_{i}=\underline{x}\right\}$ and $\left\{i \mid y_{i}=\bar{x}\right\}$ is empty. If, say, $\left\{i \mid y_{i}=\underline{x}\right\}$ is empty, then $y_{i}>\underline{x}$ for all $1 \leq i \leq n$, and furthermore

$$
\|y-P(y)\|=\frac{\max _{i} y_{i}-\min _{i} y_{i}}{2} \leq \frac{\bar{x}-\min _{i} y_{i}}{2}<\frac{\bar{x}-\underline{x}}{2}=\|x-P(x)\| ;
$$

therefore $H$ is pseudocontractive. In other words, $F\left(t_{0}+B^{\prime}\right) F\left(t_{0}+B^{\prime}-\right.$ 1) $\cdots F\left(t_{0}\right)$ is pseudocontractive for $B^{\prime}=(n-1) B$.

Let us briefly summarize what we have presented in this section. In the bidirectional case, the weight matrices are always paracontracting. Theorem 1 can be applied directly to infer the convergence of the consensus processes. In the unidirectional case, the weight matrices are generally not pseudocontracting. In order to use Theorem 2, the uniform connectivity condition needs to be imposed so that the matrix products across certain time interval are pseudocontracting.

\section{Iteration Graph and Nonlinear Asynchronous Consensus Protocols}

In the framework of [27], the consensus problem is regarded as a special case of finding common fixed points of a finite set of paracontracting multiple point operators. That is, all the operators are defined on (different) products of $\mathbb{R}^{n}$. To avoid the divergent phenomena, asynchronous iterations which fulfill 
certain coupling assumptions called confluent are considered. Below we apply this theory of paracontractions and confluence to derive a more general consensus result, which extends Result No. 9 by allowing nonlinear multiple point operators.

For the purpose of self-containedness, we introduce several related definitions below. Let $\mathbb{I}$ be a set of indices, $m \in \mathbb{N}$ a fixed number, and $\mathcal{F}=\left\{F^{i} \mid i \in\right.$ $\mathbb{I}\}$ be a pool of operators $F^{i}: D^{m_{i}} \subset \mathbb{R}^{n m_{i}} \rightarrow D$, where $m_{i} \in\{1, \cdots, m\}$, $\forall i \in \mathbb{I}$, and $D \subset \mathbb{R}^{n}$ is closed. Furthermore, let $\mathcal{X}_{\mathcal{O}}=\{x(0), \cdots, x(-M)\} \subset D$ be a given set of vectors. Then, for sequences $\mathcal{I}=I(k)(k=0,1, \ldots)$ of elements in $\mathbb{I}, \mathcal{S}=\left\{s^{1}(k), \ldots, s^{m_{i(k)}}(k)\right\}, k=0,1, \ldots$, of $m_{i}$-tuple from $\mathbb{N}_{0} \cup\{-1, \ldots,-M\}$ with $s^{l}(k) \leq k$ for all $k \in \mathbb{N}_{0}, l=1, \ldots, m_{i(k)}$, we study the asynchronous iteration given by

$$
x(k+1)=F^{I(k)}\left(x\left(s^{1}(k)\right), \ldots, x\left(s^{m_{I(k)}}(k)\right)\right), k=0,1, \ldots
$$

An asynchronous iteration corresponding to $\mathcal{F}$, starting with $\mathcal{X}_{\mathcal{O}}$ and defined by $\mathcal{I}$ and $\mathcal{S}$ can be denoted by $\left(\mathcal{F}, \mathcal{X}_{\mathcal{O}}, \mathcal{I}, \mathcal{S}\right)$. A fixed point $\xi$ of a multiple point operator $F: \mathbb{R}^{n m} \rightarrow \mathbb{R}$ is a vector $\xi \in \mathbb{R}^{n}$ which satisfies $F(\xi, \ldots, \xi)=\xi$, and a common fixed point of a pool is a fixed point of all its operators in this sense.

\subsection{Iteration Graph}

In essence, the communication assumptions define the coupling among agents or, more generally, the coupling of an iteration process. The existing assumptions often rely on interaction graphs to describe the "spatial" coupling among agents. However, ambiguity arises when asynchronism (e.g., delays) is allowed since the "temporal" coupling cannot be described directly. In the asynchronous setting, it is of importance to differentiate the same agent at different time instants.

To this end, we associate an iteration graph with the asynchronous iteration $\left(\mathcal{F}, \mathcal{X}_{\mathcal{O}}, \mathcal{I}, \mathcal{S}\right)$. Every iteration, including initial vectors, gets a vertex, so the set of vertices is $V=\mathbb{N}_{0} \cup\{-1, \ldots,-M\}$. A pair $\left(k_{1}, k_{2}\right)$ is an element of the set of edges $E$ in the iteration graph $(V, E)$, if and only if the $k_{1}$ th iteration vector is used for the computation of the $k_{2}$ th iteration vector.

Below we illustrate the concept of iteration graph via an example. The interaction topologies of a three-agent system at different time instants are shown in Fig. 2(a). It is easy to see that if the interaction pattern continues, Assumption 3(b) is satisfied. Let $y(-1)=x_{1}(0), y(-2)=x_{2}(0)$, and $y(-3)=$ $x_{3}(0)$. At time $k=0, v_{2}$ communicates with $v_{3}$. By construction, we add a vertex 0 and an edge from vertex -2 to vertex 0 in the associated iteration graph, as shown in Fig. 2(b). Assume that $v_{3}$ and $v_{1}$ do not use its own past value. Therefore, we do not add an edge from vertex -3 to vertex 0 in the iteration graph. At time instant $k=1, v_{2}$ uses the value of $v_{1}$ and its own past value to update its state, resulting in two edges in the iteration graph. 
Remark 1. An analogy to the iteration graph is the the reachability graph in the Petri net literature. The reachability graph is used for verification and supervisory control and obtained sometimes via a method called unfolding that simplifies the procedure [4].
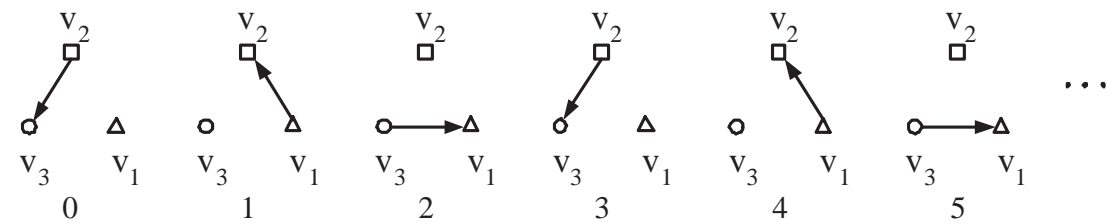

(a)

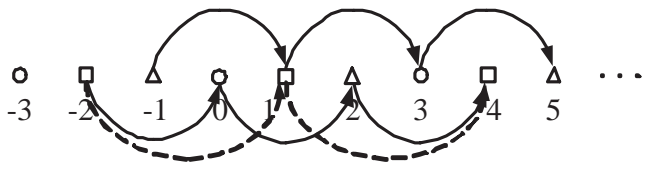

(b)

Fig. 2. Interaction topologies of an asynchronous system and its associated iteration graph.

Definition 1 (Confluent asynchronous iteration [27]). Let $\left(\mathcal{F}, \mathcal{X}_{\mathcal{O}}, \mathcal{I}, \mathcal{S}\right)$ be an synchronous iteration. The iteration graph of $\left(\mathcal{F}, \mathcal{X}_{\mathcal{O}}, \mathcal{I}, \mathcal{S}\right)$ is the digraph $(V, E)$, whose vertices $V$ are $\mathbb{N}_{0} \cup\{-1, \ldots,-M\}$, and whose edges $E$ are given by

$$
\left(k, k_{0}\right) \in E \text {, iff there is an } 1 \leq l \leq m_{I\left(k_{0}-1\right)} \text {, such that } s^{l}\left(k_{0}-1\right)=k .
$$

$\left(\mathcal{F}, \mathcal{X}_{\mathcal{O}}, \mathcal{I}, \mathcal{S}\right)$ is called confluent, if there are numbers $n_{0} \in \mathbb{N}, b \in \mathbb{N}$ and $a$ sequence $b_{k}\left(k=n_{0}, n_{0}+1, \ldots\right)$ in $\mathbb{N}$, such that for all $k \geq n_{0}$ the following is true:

(i) For every vertex $k_{0} \geq k$ there is a directed path from $b_{k}$ to $k_{0}$ in $(V, E)$,

(ii) $k-b_{k} \leq B$,

(iii) $\mathcal{S}$ is regulated,

(iv) for every $i \in \mathbb{I}$ there is a $c_{i} \in \mathbb{N}$ so that for all $k \geq n_{0}$ there is a vertex $w_{k}^{i}$ in $V$, which is a successor of $b_{k}$ and a predecessor of $b_{k+c_{i}}$, and for which is $I\left(w_{k}^{i}-1\right)=i$.

It is worth mentioning that when Assumptions 2(a)(b), 3(b), 4(b) are fulfilled, the associated asynchronous iteration is confluent. Given an arbitrary iteration, there are simple ways to make its implementation confluent [27].

Remark 2. As opposed to the original development in [9], here the whole vector is updated in every iteration step. Also, all components of vectors have the 
same delay. This does not impose a restriction since the vectors reduce to a scalar in our study.

\subsection{Nonlinear Asynchronous Consensus Protocols}

Before introducing the main result of the paper, we need the following definition. Note that paracontracting operator in Definition 2(ii) corresponds to pseudocontracting operator as defined in (3). (Without much confusion, the original definitions given in [27] are followed for easy reference.)

Definition 2. Let $\mathcal{F}$ be a pool of operations as in Definition 1, and $X=$ $\left(x^{1}, \ldots, x^{m_{i}}\right)$ an element of $\mathbb{R}^{n m_{i}}$.

(i) If for all $i \in \mathbb{I}, X, Y \in D^{m_{i}}$ and a norm $\|\cdot\|$

$$
\left\|G^{i}(X)-G^{i}(Y)\right\|<\max _{j}\left\|x^{j}-y^{j}\right\|
$$

or $\left\|G^{i}(X)-G^{i}(Y)\right\|=x^{j}-y^{j}, \forall j \in\left\{1, \ldots, m_{i}\right\}$, then $\mathcal{F}$ is called strictly nonexpansive on $D$.

(ii) If for all $i \in \mathbb{I}, X \in D^{m_{i}}$ and a norm $\|\cdot\|, F^{i}$ is continuous on $D^{m_{i}}$, then $\mathcal{F}$ is paracontracting on $D$, if for any fixed point $\xi \in \mathbb{R}^{n}$ of $F^{i}$,

$$
\left\|F^{i}(X)-\xi\right\|<\max _{j}\left\|x^{j}-\xi\right\|
$$

or $X=(x, \ldots, x)$ and $x$ is a fixed point of $F^{i}$.

It is easy to see that every strictly nonexpansive pool is paracontracting. Moreover, an (e=extended)-paracontracting notion is introduced in [27]. The (e)-paracontracting operators may be discontinuous, and have nonconvex sets of fixed points.

A simplified version of the main result in [27] is now given.

Theorem 3. Let $\mathcal{F}$ be a paracontracting pool on $D \subset \mathbb{R}^{n}$, and assume that $\mathcal{F}$ has a common fixed point $\xi \in D$. Then any confluent asynchronous iterations $\left(\mathcal{F}, \mathcal{X}_{\mathcal{O}}, \mathcal{I}, \mathcal{S}\right)$ converges to a common fixed point of $\mathcal{F}$.

With the help of Theorem 3, Result No. 9 can be obtained by interpreting the different rows of a stochastic matrix as multiple data operators. To see this, let $f_{i m_{i}(j)}(k)$ be for all $i \in\{1, \ldots, n\}$ the $j$ th of $m_{i}$ nonzero entries in $F(k)$ 's $i$ th row, or let $m_{i}(j)=j, \forall j=1, \ldots, n$, and $m_{i}=n$, if this row is zero. Then the pool $\mathcal{F}=\left\{F^{i} \mid i=1, \ldots, Q\right\}(Q$, the total number of operators, is finite), defined by $F^{i}(k): \mathbb{R}^{m_{i}} \rightarrow \mathbb{R}$,

$$
F^{i}(k)\left(y^{1}, \ldots, y^{m_{i}}\right):=\sum_{j=1}^{m_{i}} f_{i m_{i}(j)}(k) y^{j}, i=1, \ldots, Q
$$

is strictly nonexpansive on all closed intervals $D \subset \mathbb{R}$, if $F^{i}(1, \ldots, 1)=1$.

We are now ready to claim a new consensus result where $F^{i}$ is allowed to be nonlinear. To avoid confusion, we rewrite $I(k)$ in $(12)$ as $p_{k}$ below. 
Theorem 4. Consider the iteration

$$
x_{i}(k+1)=F^{i}\left(x _ { 1 } \left(s^{1}(k), x_{2}\left(s^{2}(k)\right), \ldots, x_{n}\left(s^{n}(k)\right) .\right.\right.
$$

(i) Assume without loss of generality that the numbering of $s^{l}(k), k=0,1, \ldots$, is chosen in such a manner that all components $x_{l}\left(s^{l}(k)\right)$ in (14) themselves are updated at time $s^{l}(k)$, i.e.,

$$
p_{s^{i}(k)-1}=i, \forall k \in \mathbb{N}, i \in\{1, \ldots, n\} \text { with } s^{i}(k) \geq 1,
$$

and, also w.l.o.g., that all initial vectors are multiples of $\mathbf{1}$. Define

$$
x(-k):=x_{k}(0) \mathbf{1}, \forall k=1, \ldots, n,
$$

and renumber in this way the elements of the sequences of $s^{l}(k), k=$ $0,1, \ldots, l=1, \ldots, n$, for which $s^{l}(k)=0$. Then the asynchronous iteration $\left(\mathcal{F}, \mathcal{Y}_{\mathcal{O}}, \mathcal{I}, \mathcal{S}\right)$, given by

$$
y(k+1):=F^{p_{k}}\left(y\left(\tilde{s}^{1}(k)\right), \ldots, y\left(\tilde{s}^{m_{p_{k}}}(k)\right)\right), k=0,1, \ldots
$$

where $\mathcal{F}=\left\{F^{p_{k}} \mid k=0,1, \ldots\right\}$ is paracontracting, $\mathcal{I}=p_{k}, k=0,1, \ldots$, $\mathcal{S}=\left\{\tilde{s}^{i}(k) \mid k=0,1, \ldots ; i=1, \ldots, m_{p_{k}}\right\}$ is given by

$$
\tilde{s}^{i}(k):=s^{m_{p_{k}}(i)}(k), \forall k \in \mathbb{N}_{0}, i=1, \ldots, m_{p_{k}},
$$

and $\mathcal{Y}_{O}$ by $y(-l):=x_{1}(-l), l=1, \ldots, n$, generates

$$
y(k+1)=x_{p_{k}}(k+1), \forall k \in \mathbb{N}_{0} .
$$

(ii) The pool $\mathcal{F}=\left\{F^{i}: \mathbb{R}^{n} \rightarrow \mathbb{R} \mid i \in\{1, \ldots, Q\}\right\}$ is paracontracting and has a common fixed point. Furthermore, there exists an agent $i_{0}$ which updates its state using only a subset of the pool $\mathcal{F}$. Every operator in this subset is continuously differentiable in $x_{i_{0}}$ and $\frac{\partial F^{i}}{\partial x_{i_{0}}} \neq 0$. Assume that $s^{i_{0}}(k)=$ $\max \left\{k_{0} \leq k \mid p_{k_{0}-1}=i_{0}\right\}$ for all $k>\min \left\{k_{0} \in \mathbb{N}_{0} \mid p_{k_{0}}=i_{0}\right\}$ with $p_{k}=i_{0}$. Then, under Assumptions 2(a)(b), 3(b), 4(b), the nonlinear protocol (16) or, equivalently, (14) guarantees asymptotic consensus.

Proof. (i) follows by induction on $k$. Using the same argument as in the proof of Theorem 5(v) in [27], it can be shown that the iteration (16) is confluent. (ii) is then an immediate result of Theorem 3 .

Remark 3. In Fig. 2, the iteration graph is confluent when the agent $v_{2}$ always uses its own past value for updating. Suppose that no agents use their past values during the process (i.e., dashed edges no longer appear). After removing the dashed edges from Fig. 2(b), the iteration graph is no longer confluent since there is no directed path from an odd-numbered vertex to an even-numbered vertex, and vice versa. This shows the necessity of existence of $i_{0}$ in Theorem $4($ ii).

Theorem 4 is exact, rather than linearized and can be used to study multiagent systems with nonlinear couplings. Potential applications include distributed time synchronization and rendezvous of multi-robots with nonholonomic constraints. 


\section{Conclusions}

In this paper, we examined the different assumptions made in the various consensus results in the literature so to better understand their roles in the convergence analysis of consensus protocols. A novel nonlinear asynchronous consensus result was also introduced using the theory of paracontracitons and confluence. This result is more general than the existing ones and provides a powerful tool to study a wider range of applications. Many open problems remain; see [13] for a detailed discussion.

\section{References}

1. D. Bauso, L. Giarre, and R. Pesenti. Attitude alignment of a team of UAVs under decentralized information structure. In Proc. 2003 IEEE Conf. Contr. Appl., pages 486-491, Istanbul, Turkey, 2003.

2. R. W. Beard and V. Stepanyan. Information consensus in distributed multiple vehicle coordinated control. In Proc. 42nd IEEE CDC, pages 2029-2034, Maui, HI, 2003.

3. G. Beni and P. Liang. Pattern reconfiguration in swarms - convergence of a distributed asynchronous and bounded iterative algorithm. IEEE Trans. Robot. Automat., 12(3):485-490, 1996.

4. A. Benveniste, E. Fabre, S. Haar, and C. Jard. Diagnosis of asynchronous discrete-event systems: a net unfolding approach. IEEE Trans. Automat. Contr., 48(5):714-727, 2003.

5. D. P. Bertsekas and J. N. Tsitsiklis. Parallel and Distributed Computation: Numerical Methods. Prentice-Hall, Englewood Cliffs, NJ, 1989.

6. Vincent D. Blondel, Julien M. Hendrickx, Alex Olshevsky, and John N. Tsitsiklis. Convergence in multiagent coordination, consensus, and flocking. In Proc. 44th IEEE CDC and ECC05, Seville, Spain, 2005.

7. R. Bru, L. Elsner, and M. Neumann. Convergence of infinite products of matrices and inner-outer iteration schemes. Electronic Trans. Numerical Analysis, 2:183193, 1994.

8. M. Cao, A. S. Morse, and B. D. O. Anderson. Coordination of an asynchronous multi-agent system via averaging. In 16th IFAC World Congress, Prague, July 4-8 2005 .

9. D. Chazan and W. Miranker. Chaotic relaxation. Lin. Algebra and Its Applicat., 2:199-222, 1969.

10. G. A. de Castro and F. Paganini. Convex synthesis of controllers for consensus. In Proc. 2004 ACC, pages 4933-4938, Boston, MA, 2004.

11. L. Elsner, I. Koltracht, and M. Neumann. On the convergence of asynchronous paracontractions with applications to tomographic reconstruction from imcomplete data. Linear Algebra Appl., 130:65-82, 1990.

12. L. Fang and P. J. Antsaklis. Information consensus of asynchronous discretetime multi-agent systems. In Proc. ACC 2005, pages 1883-1888, Portland, OR, 2005.

13. Lei Fang, Panos J. Antsaklis, and Anastasis Tzimas. Asynchronous consensus protocols: preliminary results, simulations and open questions. In Proc. 44th IEEE CDC and ECC'05, Seville, Spain, 2005. 
14. A. Frommer and D. B. Szyld. On asynchronous iterations. J. Computational and Applied Math., 123:201-216, 2000.

15. Y. Hatano and M. Mesbahi. Agreement over random networks. In Proc. 43rd IEEE CDC, pages 2010-2015, Atlantis, Paradise Island, Bahamas, 2004.

16. R. A. Horn and C. R. Johnson. Matrix Analysis. Cambridge University Press, New York, 1985.

17. A. Jadbabaie, J. Lin, and A. S. Morse. Coordination of groups of mobile autonomous agents using nearest neighbor rules. IEEE Trans. Automat. Contr., 48(6):988-1001, 2003.

18. V. S. Kozyakin. Asynchronous systems: a short survey and problems, 2003.

19. S. Li and H. Wang. Multi-agent coordination using nearest neighbor rules: Revisiting the vicsek model, Jul. 2004.

20. J. Lin, A. S. Morse, and B. D. O. Anderson. The multi-agent rendezvous problem - the asynchronous case. In Proc. 43rd IEEE CDC, pages 1926-1931, Atlantis, Paradise Island, Bahamas, 2004.

21. Z. Lin, M. Broucke, and B. Francis. Local control strategies for groups of mobile autonomous agents. IEEE Trans. Automat. Contr., 49(4):622-629, 2004.

22. Y. Liu, K. Passino, and M. Polycarpou. Stability analysis of m-dimensional asynchronous swarms with a fixed communication topology. In Proc. 2002 ACC, pages 1278-1283, Anchorage, AK, 2002.

23. M. Mehyar, D. Spanos, J. Pongsajapan, S. H. Low, and R. M. Murray. Distributed averaging on asynchronous communication networks. In Proc. 44th IEEE $C D C$ and $E C C^{\prime} 05$, Seville, Spain, 2005.

24. L. Moreau. Leaderless coordination via bidirectional and unidirectional timedependent communication. In Proc. 42nd IEEE CDC, pages 3070-3075, Maui, HI, 2003.

25. L. Moreau. A note on leaderless coordination via bidirectional and unidirectional time-dependent communication. In Proc. MTNS'04, Leuven, Belgium, 2004.

26. R. Olfati-Saber and R. M. Murray. Consensus problems in networks of agents with switching topology and time-delays. IEEE Trans. Automat. Contr., 49(4):1520-1533, 2004.

27. M. Pott. On the convergence of asynchronous iteration methods for nonlinear paracontractions and consistent linear systems. Lin. Algebra and Its Applicat., 283:1-33, 1998 .

28. W. Ren and R. W. Beard. Consensus seeking in multiagent systems under dynamically changing interaction topologies. IEEE Trans. Automat. Contr., 50(5):655-661, 2005.

29. W. Ren, R. W. Beard, and E. M. Atkins. A survey of consensus problems in multi-agent coordination. In Proc. ACC 2005, pages 1859-1864, Portland, OR, 2005 .

30. W. Ren, R. W. Beard, and T. W. McLain. Coordination variables and consensus building in multiple vehicle systems. In V. Kumar, N. leonard, and A. S. morse, editors, Cooperative Control, volume 309 of LNCIS, pages 171-188. Springer, Berlin Heidelberg, 2004.

31. J. J. E. Slotine and W. Wang. A study of synchronization and group cooperation using partial contraction theory. In V. J. Kumar, N. E. Leonard, and A. S. Morse, editors, Cooperative Control, volume 309 of LNCIS, pages 207-228. Springer, Berlin Heidelberg, 2003. 
32. Yangfeng Su and Amit Bhaya. Convergence of pseudocontractions and applications to two-stage and asynchronous multisplitting for singular M-matrices. SIAM J. Matrix Anal. Appl., 22(3):948-964, 2001.

33. J. N. Tsitsiklis, D. P. Bertsekas, and M. Athans. Distributed asynchronous deterministic and stochastic gradient optimization algorithms. IEEE Trans.Automat. Contr., 31(9):803-812, 1986.

34. F. J. Vazquez-Abad, C. G. Cassandras, and V. Julka. Centralized and decentralized asynchronous optimization of stochastic discrete-event systems. IEEE Trans. Automat. Contr., 43(5):631-655, 1998.

35. L. Xiao and S. Boyd. Fast linear iterations for distributed averaging. In Proc. 42nd IEEE CDC, pages 4997-5002, Maui, HI, 2003.

36. L. Xiao, S. Boyd, and S. Lall. A scheme for robust distributed sensor fusion based on average consensus. In IPSN'05, pages 63-70, Los Angeles, CA, 2005. 